\title{
Changes in Lens Power in Singapore Chinese Children during Refractive Development
}

\author{
Rafael Iribarren, ${ }^{1}$ Ian G. Morgan, ${ }^{2}$ Yiong Huak Chan, ${ }^{3}$ Xiaoyu Lin, ${ }^{4}$ and Seang-Mei Saw ${ }^{4}$
}

Purpose. To examine changes in lens power during refractive development in Singapore Chinese children.

Methods. Children aged six to nine years from three Singapore schools were invited to participate in the Singapore Cohort study of the Risk factors for Myopia (SCORM) study. Cycloplegic refractions and biometry were measured annually in the schools over a five year period from 1999. Children were classified into five refractive error groups: persistent hyperopia, emmetropizing hyperopia, persistent emmetropia, newly developed myopia, or persistent myopia. Crystalline lens power was calculated using Bennett's formula. The rate of change per year across the refractive groups was adjusted for age and sex using General Linear Models.

REsults. There were 1747 children with at least three sets of measurements for lens power calculations. The mean age at baseline was $7.94 \pm 0.84$ years and the mean spherical equivalent refraction was $-0.41 \pm 1.71$ diopters (D). Lower lens power and lower lens thickness were associated with persistent myopia. As expected, the newly developed myopes and the persistent myopes showed the largest changes in axial length (AL). Changes in lens power and thickness at follow-up were similar in all refractive groups, except for the newly developed myopes, who showed significantly greater decreases in lens power (0.36 vs. $0.29 \mathrm{D} /$ year; $P<0.001)$ and lens thickness $(0.015$ vs. $0.0003 \mathrm{~mm} /$ year; $P<0.001)$ than the persistently emmetropic group.

Conclusions. Newly developed myopes showed a significantly greater decrease in lens power than other refractive groups, which may be linked to rapid changes in AL and refraction that occur around the onset of myopia. (Invest Ophthalmol Vis Sci. 2012;53:5124-5130) DOI:10.1167/iovs.12-9637

From the ${ }^{1}$ Department of Ophthalmology, Centro Médico San Luis, Buenos Aires, Argentina; The ${ }^{2}$ Australian Research Council (ARC) Centre of Excellence in Vision Science, Research School of Biology, College of Medicine, Biology and Environment, Australian National University, Canberra, Australia; the ${ }^{3}$ Biostatistics Unit, Yong Loo Lin School of Medicine, National University Health System, Singapore; and the "Saw Swee Hock School of Public Health, National University of Singapore.

Supported by grants from the Singapore Cohort Of the Risk factors for Myopia (SCORM) from the National Medical Research Council, Singapore (NMRC/0975/2005); and the Australian Research Council Centre of Excellence in Vision Science (CEO561903 [IGM]).

Submitted for publication February 5, 2012; revised May 25, June 15, and June 30, 2012; accepted July 3, 2012.

Disclosure: R. Iribarren, None; I.G. Morgan, None; Y.H. Chan, None; X. Lin, None; S.-M. Saw, None

Corresponding author: Rafael Iribarren, Department of Ophthalmology, Centro Médico San Luis, San Martín de Tours 2980, (1425) Buenos Aires, Argentina; rafairibarren@gmail.com.
S pherical equivalent refraction (SER) is determined by the $\checkmark$ balance of corneal and lens power, anterior chamber depth (ACD), and axial length (AL). In newborn children, ${ }^{1}$ the distribution of refractive error is normal, and centered around a mean hyperopic status of 2 to 3 diopters (D). Changes in the first year or two after birth show marked increases in AL and decreases in corneal and lens power, coupled with the development of a markedly kurtotic distribution of SER, with a peak of around $1 \mathrm{D}$, and a corresponding reduction in the variability of SER. ${ }^{2-4}$

The classical cross-sectional and longitudinal studies by Sorsby and colleagues ${ }^{5,6}$ of changes in refraction after the age of 3 define the next stage; increases in AL and ACD, with very limited changes in corneal power, but significant reductions in lens power. The changes in refraction were smaller than anticipated from the amount of axial elongation, suggesting that the reductions in lens power largely neutralized the myopic shifts, which would otherwise be associated with axial elongation..$^{7,8}$

These changes in lens power are potentially important for the determination of final refractive state. Refraction is clustered by emmetropisation around a mean SER of about $+1 \mathrm{D}$ in the first two years of life, and from then on there is an increase in AL of at least $3 \mathrm{~mm}$ up to adult values, which corresponds to a myopic shift in refraction of around 6 to $7 \mathrm{D}^{8}$ Without a compensating factor, most children would, therefore, become highly myopic. The major compensating factor appears to be reduction in lens power, but despite its importance, surprisingly little attention has been devoted to the mechanisms of change in lens power or how lens power is controlled during development.

Most previous studies have been carried out on populations where the prevalence of myopia is relatively low, as is the case for the Orinda ${ }^{9}$ and Collaborative Longitudinal Evaluation of Ethnicity and Refractive Error (CLEERE) ${ }^{10}$ studies. A previous report from the Singapore Cohort study of the Risk factors for Myopia (SCORM) study, ${ }^{11}$ where the prevalence of myopia is high, has reported on the patterns of longitudinal change in refraction and ocular biometry. The high prevalence and incidence of myopia in Singapore enabled an analysis of the differences between those children who were myopic at first visit, and those who became myopic during the follow-up period. Key findings from this previous report ${ }^{11}$ were that the rate of axial elongation was markedly higher in children who were always myopic or who became myopic than in other groups, and that changes in lens thickness were biphasic in all refractive groups.

Changes in lens power were not analyzed in the previous report. In this paper, we have used the longitudinal data on refraction and ocular components in these Singapore Chinese children to examine changes in lens power in this population where the prevalence of myopia is high.

\section{Methods}

Children aged 6 to 9 years from three schools in Singapore were invited to join SCORM in 1999 and 2001. Two schools were recruited in 1999, 
TABLE 1. Means of Ocular Components by Sex at Baseline

\begin{tabular}{lrcc}
\hline & Males & Females & $\boldsymbol{P}$ \\
\hline Spherical equivalent (diopters) & -0.51 & -0.30 & $0.013^{*}$ \\
Corneal power (diopters) & 42.51 & 43.18 & $0.001^{*}$ \\
Anterior chamber depth (mm) & 3.65 & 3.56 & $0.001^{*}$ \\
Lens power (diopters) & 24.22 & 25.39 & $0.001^{*}$ \\
Axial length (mm) & 23.62 & 23.02 & $0.001^{*}$ \\
Lens thickness (mm) & 3.47 & 3.47 & 0.886 \\
AL/CR & 3.027 & 2.997 & $0.001^{*}$ \\
\hline
\end{tabular}

*Student's $t$-test.

and one in 2001. The detailed methodology has been previously published. ${ }^{11-13}$ The study was approved by the ethics committee of the Singapore Eye Research Institute and the study protocol adhered to the tenets of the Declaration of Helsinki. Only children with written informed consent from their parents participated in the study.

The children were examined yearly in the schools by a trained team using standard methodology. After cycloplegia ( $0.5 \%$ proparacaine, followed by 3 drops of $1 \%$ cyclopentolate at 5 minute intervals), refraction and corneal radius were measured using an autokeratorefractor (model RK5; Canon Inc, Ltd., Tochigiken, Japan). An Echoscan (US-800; Nidek Co., Ltd, Tokyo, Japan) was used to measure AL, vitreous chamber depth, $\mathrm{ACD}$, and lens thickness.

\section{Definitions}

The spherical equivalent refraction of the randomly selected eye was calculated as sphere power $+(0.5 \times$ cylinder power $)$. A child had persistent hyperopia if the SER was more than $+1.00 \mathrm{D}$ at all visits. A child who began with hyperopia (SER more than $+1.00 \mathrm{D}$ ) on at least the first visit, but had SE between greater than $-0.50 \mathrm{D}$ and $+1.00 \mathrm{D}$ at subsequent visits, was considered to have emmetropizing hyperopia. A child was deemed to have persistent emmetropia if the SER was greater than $-0.50 \mathrm{D}$ and less than or equal to $+1.00 \mathrm{D}$ at all visits. A child who began with emmetropia on at least the first visit and demonstrated a SER lower than $-0.50 \mathrm{D}$ at one or more subsequent visits was classified as having newly developed myopia. Persistent myopia was defined by a SER of at least $-0.50 \mathrm{D}$ at all visits.

There were 1747 children at baseline and the retention rate was $97.3 \%, 98.3 \%, 90.9 \%, 62.3 \%$, and $34.7 \%$ for the five subsequent visits. All children who had at least three visits and met the criteria for one of the five refractive error groups, were included in the analyses.

Corneal power, in diopters, was calculated from the corneal radius with a refractive index of 1.3315 , as proposed by Olsen. ${ }^{14}$ Calculation of crystalline lens power was based on distance, cycloplegic autorefraction, corneal power, ACD, lens thickness, and AL using the formula proposed by Bennett. ${ }^{15}$ For the calculation of the A and B constants of this formula, a $Q$ value of 0.36 was used $^{15}$ and a lens equivalent index of 1.427 was used, as proposed by Mutti for children's lenses. ${ }^{16}$

\section{Statistical Analysis}

The mean values for ocular components were compared at baseline across the refractive groups after age-sex and multivariate adjustment. For the multivariate adjusted models, potential risk factors were included in multivariate regression models, and manual backward stepwise elimination procedures were performed, based on a criterion of $P$ less than 0.05 , to achieve the most parsimonious model. Simple correlations were studied between the different ocular components and refraction with Pearson $r$ values. Stepwise multiple linear regression analysis with collinearity diagnostics was performed for refractive error, including the ocular components that were significantly correlated with spherical equivalent.

Growth curve models of AL, ACD, lens thickness, and corneal radius were presented in a previous publication. ${ }^{11}$ This paper shows the fractional polynomial curves only for lens power change, obtained using the same methods. The rate of change per year across the refractive groups was then studied for spherical equivalent refraction, AL, lens power, and lens thickness (unadjusted, age adjusted, sex adjusted, and multivariate adjusted). The variables included in multivariate adjustment were age, sex, race, father's education, school, height at baseline visit, parental myopia, and books read per week (considered as fixed effects). ${ }^{12,13,17}$ Data analyses were performed with statistical software (SPSS version 19.0; SPSS Inc., Chicago, IL).

TABLE 2. Mean Values for Ocular Components at Baseline Visit across the Refractive Groups

\begin{tabular}{|c|c|c|c|c|c|c|}
\hline$n=1747$ & $\begin{array}{c}\text { PH } \\
46\end{array}$ & $\begin{array}{c}\text { EH } \\
141\end{array}$ & $\begin{array}{c}\text { PE } \\
361\end{array}$ & $\begin{array}{c}\text { NDM } \\
590\end{array}$ & $\begin{array}{l}\text { PM } \\
609\end{array}$ & $P^{*}$ \\
\hline \multicolumn{7}{|c|}{ Lens Power (diopters), Mean (SD) } \\
\hline Unadjusted & $25.53(1.72)$ & $25.52(1.53) \dagger$ & $25.07(1.52)$ & $25.04(1.61)$ & $24.17(1.59) \dagger$ & $<0.001$ \\
\hline Age-sex adjusted & $25.46(1.41)$ & $25.29(1.42)$ & $25.26(1.42)$ & $24.86(1.44) \dagger$ & $24.31(1.43) \dagger$ & $<0.001$ \\
\hline Multivariate adjusted $\neq$ & $25.63(1.53)$ & $25.45(1.76)$ & $25.42(2.23)$ & $25.07(2.68) \dagger$ & $24.5(2.71) \dagger$ & $<0.001$ \\
\hline \multicolumn{7}{|c|}{ Spherical Equivalent (diopters), Mean (SD) } \\
\hline Unadjusted & $+2.24(1.24) \dagger$ & $+1.28(0.26) \dagger$ & $+0.58(0.28)$ & $+0.24(0.37) \dagger$ & $-2.21(1.62) \dagger$ & $<0.001$ \\
\hline Age-sex adjusted & $+2.22(1.00) \dagger$ & $+1.21(1.00) \dagger$ & $+0.63(1.00)$ & $+0.18(1.01) \dagger$ & $-2.16(1.00) \dagger$ & $<0.001$ \\
\hline Multivariate adjusted $\neq$ & $+2.16(1.08) \dagger$ & $+1.14(1.25) \dagger$ & $+0.54(1.58)$ & $+0.11(1.9) \dagger$ & $-2.23(1.92) \dagger$ & $<0.001$ \\
\hline \multicolumn{7}{|c|}{ Axial Length (mm), Mean (SD) } \\
\hline Unadjusted & $22.32(0.80) \dagger$ & $22.54(0.69) \dagger$ & $23.00(0.68)$ & $23.06(0.69)$ & $24.04(0.92) \dagger$ & $<0.001$ \\
\hline Age-sex adjusted & $22.35(0.70) \dagger$ & $22.64(0.7) \dagger$ & $22.9(0.71)$ & $23.14(0.71) \dagger$ & $23.98(0.71) \dagger$ & $<0.001$ \\
\hline Multivariate adjusted $\neq$ & $22.33(0.74) \dagger$ & $22.62(0.86) \dagger$ & $22.87(1.08)$ & $23.08(1.3) \dagger$ & $23.93(1.31) \dagger$ & $<0.001$ \\
\hline \multicolumn{7}{|c|}{ Lens Thickness (mm), Mean (SD) } \\
\hline Unadjusted & $3.52(0.16)$ & $3.50(0.18)$ & $3.47(0.18)$ & $3.49(0.18)$ & $3.44(0.17)$ & $<0.001$ \\
\hline Age-sex adjusted & $3.51(0.18)$ & $3.49(0.18)$ & $3.47(0.18)$ & $3.48(0.18)$ & $3.45(0.18)$ & 0.002 \\
\hline Multivariate adjusted $\neq$ & $3.52(0.19)$ & $3.48(0.22)$ & $3.47(0.28)$ & $3.48(0.33)$ & $3.44(0.34)$ & $<0.001$ \\
\hline
\end{tabular}

${ }^{*} P$ value for one way ANOVA or General Linear Models, as appropriate.

†Significant difference when compared with PE by Bonferrroni post hoc tests.

$¥$ Multivariate GLM adjusted for age, sex, race, father’s education, school, height, parental myopia, and books read per week. 


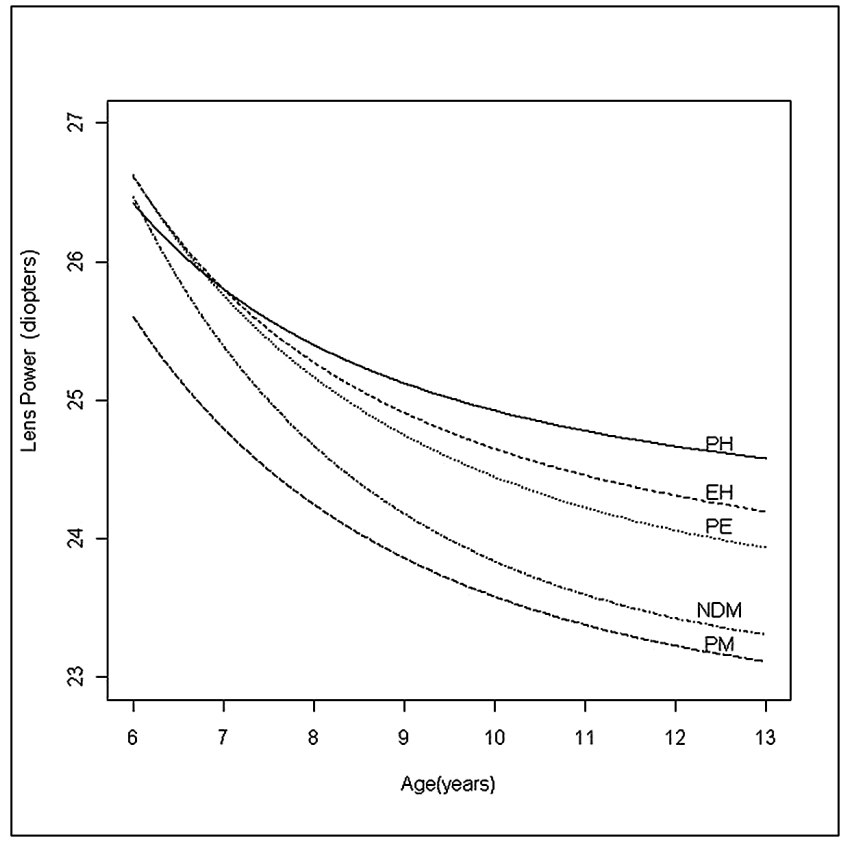

Figure 1. Sex adjusted fractional polynomial curves of lens power, in diopters, during follow-up period for the five different refractive groups: persitent hyperopes $(\mathrm{PH})$, emmetropizing hyperopes $(\mathrm{EH}), \mathrm{PE}$, NDM, and PM.

\section{Results}

There were 1747 children, with at least three visits, available for lens power calculations. At baseline, school children were from grades first to third, aged six to nine years $(72.64 \%$ were aged seven or eight years), and 887 (50.8\%) were male. The mean age at baseline was $7.94 \pm 0.84$ years and the SER was $-0.41 \pm 1.71 \mathrm{D}$. Compared with boys, girls had a significantly higher (less myopic) mean SER, greater corneal power, shorter $\mathrm{AL}$ and $\mathrm{ACD}$, lower axial length/corneal radius ratio (AL/CR), and greater lens power, but there was no difference in lens thickness between sexes (Table 1).

At baseline, unadjusted data showed that persistent myopes (PM) had lower mean lens power than emmetropes, but lens power in the newly developed myopes (NDM) was not statistically different from that of emmetropes. When adjusted for sex and age, both NDM and PM had significantly lower lens power than persistent emmetropes (PE) (Table 2), although the difference between NDM and emmetropes was small. The NDM, although emmetropic at baseline, also had lower SER and greater AL than PE.

The changes in lens power with age in the different refractive groups are shown in Figure 1. In all refractive groups, the fractional polynomial cruves ${ }^{11}$ showed very similar reductions in lens power with age. The reductions were particularly marked over the age from 6 to around 10 years. After 10 years of age, the rate of loss of lens power decreased markedly. Interestingly, there was a greater loss of lens power in those who became myopic during the follow-up period, such that, although they began with a lens power that was similar to that of the PE, at the end of that period, their lens power was closer to that of the persistent myope group.

The correlations between refraction and ocular components at baseline are shown in Table 3. As expected, SER was strongly correlated with AL, and even more strongly with the $\mathrm{AL} / \mathrm{CR}$ ratio. In contrast, the positive correlation of SER with lens power was only moderate, as shown in Figure $2 \mathrm{~A}$. The strongest correlation was seen for lens power and AL (Table 2), and the correlation between lens power and AL/CR ratio was slightly lower. The correlation between lens power and thickness was positive (Fig. 2B), showing that thinner lenses had lower lens power. Stepwise multiple linear regression analysis with spherical equivalent refractive error as the dependent variable showed $\mathrm{AL}$ as the principal significant independent variable $\left(r^{2}=0.488\right)$, then corneal power $\left(r^{2}=\right.$ $0.286)$, lens power $\left(r^{2}=0.171\right)$, and anterior chamber depth $\left(r^{2}=0.038\right.$; all $\left.P<0.001\right)$. When lens power was treated as the dependent variable, AL was the principal significant factor $\left(r^{2}\right.$ $=0.498)$, followed by lens thickness $\left(r^{2}=0.099\right)$, and spherical equivalent $\left(r^{2}=0.065\right.$; all $\left.P<0.001\right)$.

With follow-up, the changes in lens power and lens thickness were similar in all groups, except for the NDM who showed greater decreases in lens thickness than the PE (Table 4). NDM also had a significantly greater loss of lens power than persistent myopes (PM) $(-0.34 \mathrm{D} /$ year vs. $-0.26 \mathrm{D} /$
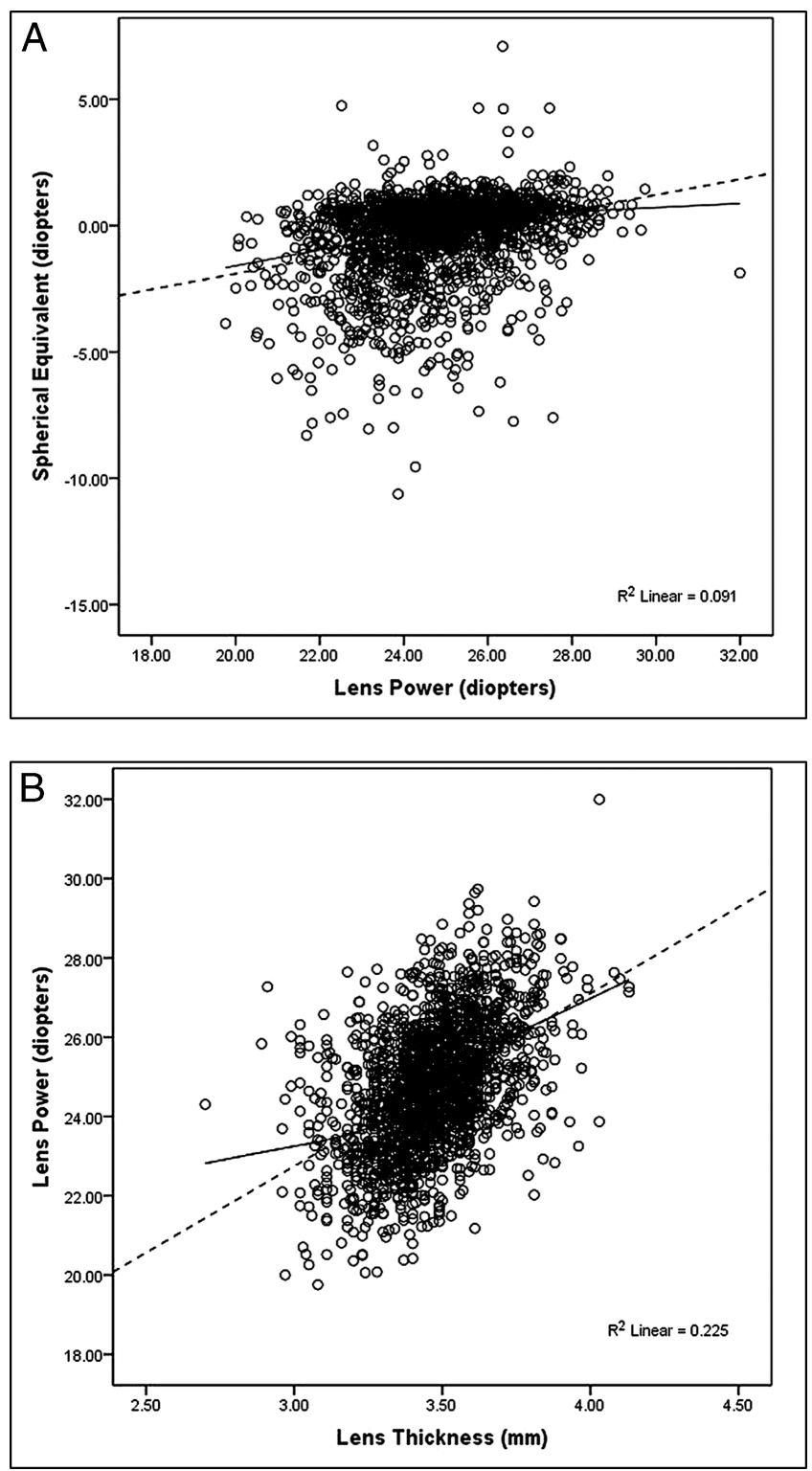

Figure 2. (A) Relationship between SER and lens power at baseline (loess plot continuous line, linear regression dotted line). (B) Relationship between lens power and lens thickness (loess plot). 
Table 3. Correlation Coefficients between Ocular Components for Baseline Data All Subjects Aged Six to 9 Years

\begin{tabular}{|c|c|c|c|c|c|}
\hline & Corneal Power & $\mathbf{A L}$ & Lens Power & Lens Thickness & AL/CR \\
\hline Refraction (SER) & $-0.107^{*}$ & $-0.698^{*}$ & $+0.302^{*}$ & $+0.139^{*}$ & $+0.865^{*}$ \\
\hline Corneal power & - & $-0.507^{*}$ & +0.208 & -0.029 & $+0.302^{*}$ \\
\hline $\mathrm{AL}$ & - & - & $-0.706^{*}$ & $-0.238^{*}$ & $+0.668^{*}$ \\
\hline Lens power & - & - & - & $+0.474^{*}$ & $-0.601^{*}$ \\
\hline Lens thickness & - & - & - & - & $-0.288^{*}$ \\
\hline
\end{tabular}

$n=1747 ; r$ values.

*Correlation is significant at the 0.01 level (two tailed).

year, $P<0.001$, Bonferroni post hoc test; Table 4). PM continued to lose lens power at a similar rate as the PE (Fig. 1 and Table 4). These changes, and the development of myopia over the follow-up period, produced some changes in the correlations between variables by the end of the study. In particular the correlations of SER with AL and with AL/CR increased (from -0.698 at baseline to -0.754 at follow-up, and from -0.865 to -0.891 , respectively), while the correlation of SER and lens power decreased (from +0.302 to +0.214 ), as did the correlation of lens power with $\mathrm{AL}$ (from -0.706 to -0.639 ) and $\mathrm{AL} / \mathrm{CR}$ ratio (from -0.601 to -0.530 ).

Three critical correlations are illustrated graphically in Figure 3 (A-C). None of the relationships were completely linear, as illustrated by the fitting of both linear regression and loess plots to the figures. In the case of spherical equivalent versus AL (Fig. 3A), spherical equivalent declined more rapidly with increasing $\mathrm{AL}$ at higher ALs. In the cases of lens power versus SER (Fig. 3B) and lens power versus AL (Fig. 3C), there appear to be slower rates of loss of lens power with myopic refractive error and longer ALs. We tested the hypothesis that, at ages 6 to 12 years, rapid progressors in the persistent myopic group could have higher rates of lens power loss, but did not find any significant difference in lens power loss between rapid and slow progressors (data not shown).

\section{Discussion}

These results show systematic changes in calculated lens power over the age range from 6 to 12 years. The curves for lens power loss in all groups were relatively steep up until the age of 10, then flattened similarly, except for the newly developed myopia group. Lens power appeared to continue to decrease, but slowly, after the age of 10 years. The changes in lens power were very similar for all the refractive groups. However, for NDM, there was a greater reduction in lens power, which was accompanied by a greater reduction in lens thickness. There was also a difference between groups in lens power at baseline, where those who started with hyperopia had the highest lens powers, those who started emmetropic had somewhat lower powers, and the group that was already myopic at baseline had the lowest starting lens power. Interestingly, NDM, while still emmetropic at baseline, had slightly greater AL and lower lens power than children with persistent emmetropia (Table 2).

These patterns of change in lens power are quite similar to those reported by Jones et al. ${ }^{9}$ from the Orinda study, with the exception that they did not divide the persistent myope and newly developed myope groups. Bearing this difference in mind, the patterns of change in lens power were quite similar, and essentially occurred in parallel, with quite similar starting points and patterns of loss. The only exception was our

TABle 4. Mean Values for Rate of Change (Final-Baseline) per Year in Ocular Components across the Refractive Groups

\begin{tabular}{|c|c|c|c|c|c|}
\hline & $\begin{array}{c}\text { EH } \\
141\end{array}$ & $\begin{array}{c}\text { PE } \\
361\end{array}$ & $\begin{array}{c}\text { NDM } \\
590\end{array}$ & $\begin{array}{l}\text { PM } \\
609\end{array}$ & $P$ value* \\
\hline \multicolumn{6}{|c|}{ Rate of Change Per Year in Lens Power (diopters/year), Mean (SD) } \\
\hline Unadjusted & $-0.24(0.30)$ & $-0.29(0.30)$ & $-0.36(0.31) \dagger$ & $-0.25(0.33)$ & $<0.001$ \\
\hline Age-sex adjusted & $-0.22(0.31)$ & $-0.31(0.31)$ & $-0.34(0.32)$ & $-0.26(0.31)$ & $<0.001$ \\
\hline Multivariate adjusted $\ddagger$ & $-0.27(0.39)$ & $-0.34(0.49)$ & $-0.38(0.59)$ & $-0.29(0.60)$ & $<0.001$ \\
\hline \multicolumn{6}{|c|}{ Rate of Change Per Year in Spherical Equivalent (diopters/year), Mean (SD) } \\
\hline Unadjusted & $-0.18(0.11) \dagger$ & $-0.11(0.11)$ & $-0.53(0.26) \dagger$ & $-0.62(0.30) \dagger$ & $<0.001$ \\
\hline Age-sex adjusted & $-0.17(0.24)$ & $-0.12(0.24)$ & $-0.52(0.25) \dagger$ & $-0.63(0.25) \dagger$ & $<0.001$ \\
\hline Multivariate adjusted $\neq$ & $-0.15(0.30)$ & $-0.09(0.38)$ & $-0.48(0.46) \dagger$ & $-0.58(0.46) \dagger$ & $<0.001$ \\
\hline \multicolumn{6}{|c|}{ Rate of Change Per Year in AL (mm/year), Mean (SD) } \\
\hline Unadjusted & $0.12(0.10)$ & $0.10(0.11)$ & $0.28(0.14) \dagger$ & $0.28(0.16) \dagger$ & $<0.001$ \\
\hline Age-sex adjusted & $0.11(0.14)$ & $0.11(0.14)$ & $0.27(0.14) \dagger$ & $0.29(0.14) \dagger$ & $<0.001$ \\
\hline Multivariate adjusted $\neq$ & $0.11(0.17)$ & $0.12(0.22)$ & $0.27(0.26) \dagger$ & $0.28(0.26) \dagger$ & $<0.001$ \\
\hline \multicolumn{6}{|c|}{ Rate of Change Per Year in Lens Thickness (mm/year), Mean (SD) } \\
\hline Unadjusted & $-0.001(0.05)$ & $0.0003(0.05)$ & $-0.015(0.04) \dagger$ & $-0.005(0.05)$ & $<0.001$ \\
\hline Age-sex adjusted & $0.001(0.05)$ & $-0.001(0.05)$ & $-0.014(0.05) \dagger$ & $-0.006(0.05)$ & $<0.001$ \\
\hline Multivariate adjusted $\ddagger$ & $0.004(0.06)$ & $0.003(0.07)$ & $-0.006(0.09) \dagger$ & $0.002(0.09)$ & 0.003 \\
\hline
\end{tabular}

Rate of Change per year $=($ last visit-baseline visit $) /$ years.

${ }^{*} P$ value for one way ANOVA or General Linear Models, as appropriate.

†Significant difference when compared with PE by Bonferrroni post hoc tests.

$¥$ Multivariate GLM adjusted for age at baseline, sex, race, father's education, school, parental myopia, and books read per week. 

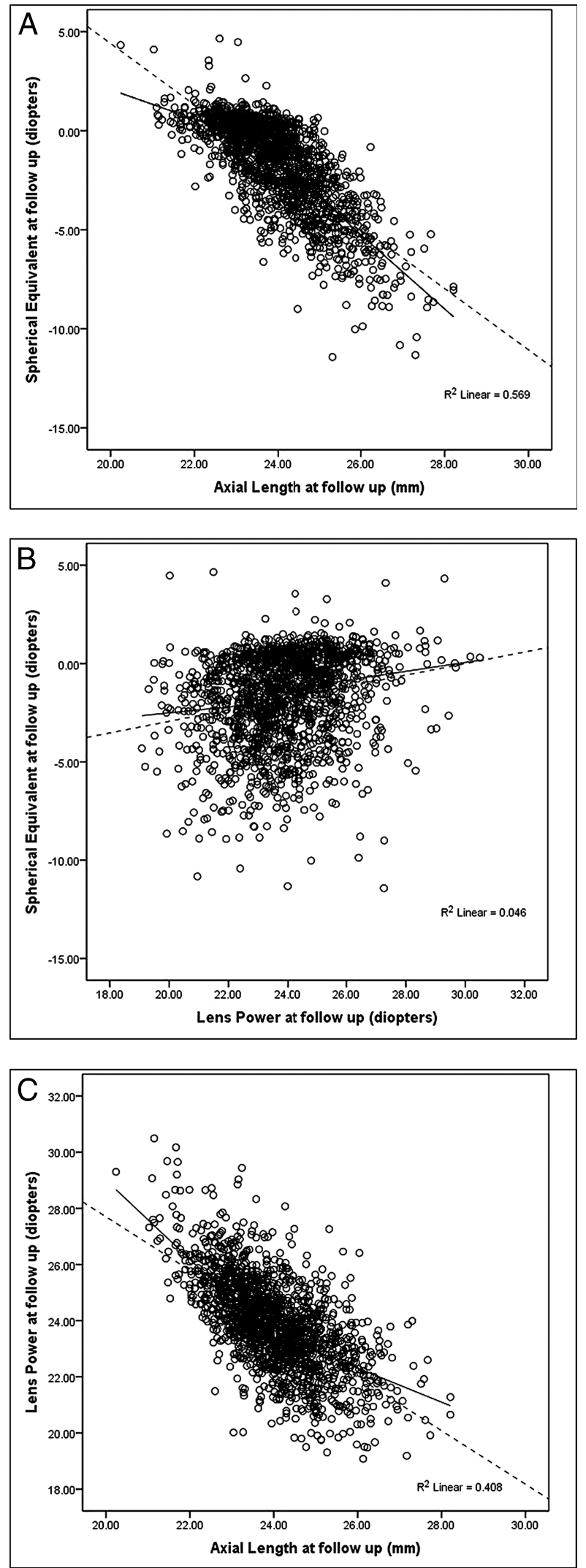

Figure 3. Loess plots for the relationships between SER and AL (A), SER and lens power (B) lens power and axial length (C) at four years follow-up. observation of a somewhat lower lens power at baseline, and greater loss of lens power at follow-up, in the newly developed myopia group. This was also reported by Garner ${ }^{18}$ in a longitudinal study of schoolchildren in Nepal, where the children developing myopia had greater reduction in lens power than their emmetropic peers.

One interpretation of these patterns of change in lens power, which appear to be similar across ethnic groups (CLEERE, ${ }^{10}$ and this study), is that they represent a developmental change that is common to most, if not all eyes, and is not regulated in relation to $\mathrm{AL}$ or refractive error. This very simple interpretation may be problematic because of the magnitude of the changes in AL and lens power that take place. If these two changes were not well-balanced, myopic shifts, or even possibly hyperopic shifts, could occur. One evolutionary solution to this problem would be to coordinate the magnitudes of natural developmental changes in the rate of axial elongation and loss of lens power, so that refractions are maintained within the mildly hyperopic range for as long as possible. This may be all that is required since the human refractive development does not lead with any precision to emmetropia. ${ }^{19}$ However, there is also convincing evidence that the rate of axial elongation in humans can be actively regulated by environmental exposures. ${ }^{20}$ Acceleration of axial elongation could then lead to a situation where axial elongation out paced reductions in lens power, or continued for longer than reduction in lens power, resulting in the development of myopia.

An alternative would be that the processes of axial elongation and loss of lens power are linked in some way. Axial elongation could be regulated in relation to the rate of loss of lens power, with any tendency to hyperopia, due to loss of lens power compensated for by increased axial elongation, ${ }^{21}$ following mechanisms that have been well documented in animal studies, ${ }^{22}$ and which appear to operate in early human refractive development. There are also some indications that loss of lens power can be accelerated, when the rate of axial elongation is high. The first comes from the baseline analysis, where there is an association between lens power and SER, and a significantly stronger association between lens power and $\mathrm{AL}$, as though in the years prior to baseline, more loss of lens power had occurred in eyes with higher rates of axial elongation. The second indication comes from analysis of the newly developed myopia group, where the process of development of myopia appears to be associated in some way with an increased loss of lens power. This would appear to be only transient, since an increased rate of loss of lens power was not detected in the follow-up analysis of the persistent myopia group, although it may have contributed to the low lens power at baseline in this group.

Other evidence suggests that the relationship between changes in lens power and axial elongation is not particularly close. Changes in lens power slow markedly after the age of 10 years, but axial elongation, while also slowing down with age, appears to outpace the rate of reduction in lens power, leading in some cases to myopia. This can be seen in the declining correlation between lens power and AL over the follow-up period. This means that the eye continues to elongate after reductions in lens power decline, making it unlikely that changes in lens power drive continuing axial elongation, and strengthening the idea that axial elongation is regulated by other factors.

Following van Alphen, ${ }^{23}$ Mutti and colleagues ${ }^{24,25}$ have suggested that the thinning of the lens, the flattening of its surfaces, and the loss of lens power are due to a simple physical stretching of the lens as the eye grows equatorially. This idea has not been critically tested, and it is worthwhile considering an alternative possibility, that the changes in lens 
thickness and power in the first 10 years of life form part of a continuing pattern of change in lens power throughout life. From the age of 10, the lens starts to thicken, while continuing to slowly lose power. This pattern continues for most of adult life, and has come to be known as the lens paradox, because of the apparent contradiction between lens thickening and increasing lens curvature occurring in parallel with loss of lens power. ${ }^{26}$ It is now generally accepted that the resolution of this paradox is that a steepening of the gradient of refractive index leads to loss of lens power. ${ }^{26-30}$

One of the features of lens development in humans is that there is a high rate of fiber formation prenatally, which slows markedly after birth. ${ }^{31}$ This may lead to a high rate of compaction of the numerous fibers laid down prenatally, combined with a reduced rate of formation of new fibers, resulting in lens thinning, flattening of lens surfaces, and loss of power over the first 10 to 12 years. After that age, as the rate of compaction of prenatal fibers declines, the slower postnatal rate of addition of new fibers could slowly thicken the ageing lens. It is possible that the compaction of the prenatal fibers in the nucleus of the lens during childhood steepens the gradient of refractive index, making the lens lose internal power. The finding of decreasing lens equivalent refractive index during childhood in the Orinda study ${ }^{9}$ can be explained by a steepening of the gradient index. And the steepening of the gradient is almost certainly related to compaction in the lens, supporting the idea that compaction between birth and age 10 produces lens thinning.

In the present study, we found lower lens power in NDM at baseline, when they were still emmetropes, and found even lower lens power in PM (Table 2). Recently, Mutti et al. ${ }^{32}$ have reported data on lens power before and after myopia onset in the CLEERE ${ }^{10}$ study. In this study they showed that lens power is lower in those who become myopic, from well before the onset of myopia, and that the difference with emmetropes becomes greater with onset. They also reported that changes in lens power ceased within a year of myopia onset, whereas lens power continued to decrease in PE. This difference in behavior between the two groups depended upon adjustment of lens power for vitreal chamber depth, but was not seen in the unadjusted data. Another recent study of the eyes of Chinese, twins before and after myopia onset, ${ }^{33}$ showed that the rate of change in SER was highest in the year prior to the first detection of myopia, with concurrent faster changes in $\mathrm{AL}$, as had been previously reported by Mutti et al., ${ }^{34}$ but that changes in lens power did not differ significantly around the onset of myopia. The differences between these three sets of results warrant further investigation, particularly since it is possible that subtle differences in the rate of lens power loss are difficult to assess.

One potential limitation of our study is the fact that lens power was calculated based on refraction, keratometry, and biometry with Bennett's formula, without phakometric measurements of lens curvatures. This formula is known to produce errors of at least $3 \mathrm{D}$ in $5 \%$ of cases, when calculating individual lens powers; however two independent studies ${ }^{35,36}$ have compared Bennett's formula against phakometric data, showing good agreement between the methods when calculating mean lens power. There is currently no method for directly measuring the power of the lens in vivo, since determining lens power from phakometric data requires assumptions about the internal gradient index.

In this study, we found a positive correlation between lens power and lens thickness. This corresponds with the evidence that both lens power and lens thickness decrease over much of this developmental period. However, some of this correlation could arise artificially because lens power was calculated using a formula that included lens thickness. We, therefore, calculated lens power with an alternative Bennett formula that does not include lens thickness. ${ }^{37}$ Using these new estimates, there was a similar significant positive correlation between lens power and measured thickness $(r=+0.313 ; P<0.001)$, showing that the correlation persists even when lens thickness is not part of the formula used to calculate lens power:

Irrespective of the mechanisms involved, this paper shows that there is a complex pattern of changes in lens thickness and power as children develop, consistent with other studies. There is a rapid loss of lens power and thickness up to the age of 10 years, with the lens slowly thickening after that age, but continuing to lose power very slowly. There is limited evidence for active regulation of the changes in the lens in relation to axial elongation and refractive development in the predominantly myopic population studied, whereas axial elongation remains the major biometric cause of myopia. But given the magnitude of the changes in lens power that take place during development, the contribution of biological variations in the rate of loss of lens power to variations in the development of refractive error needs to be assessed in more detail.

\section{References}

1. Cook RC, Glasscock RE. Refractive and ocular findings in the newborn. Am J Ophthalmol. 1951;34:1407-13.

2. Mutti DO, Mitchell GL, Jones LA, et al. Axial growth and changes in lenticular and corneal power during emmetropization in infants. Invest Ophthalmol Vis Sci. 2005;46:3074-80.

3. Mayer DL, Hansen RM, Moore BD, et al. Cycloplegic refractions in healthy children aged 1 through 48 months. Arch Ophthalmol. 2001;119:1625-8.

4. Pennie FC, Wood IC, Olsen C, et al. A longitudinal study of the biometric and refractive changes in full-term infants during the first year of life. Vision Res. 2001;41:2799-810.

5. Sorsby YA, Benjamin B, Sheridan M, et al. Refraction and its components during the growth of the eye from the age of three. Memo Med Res Counc. 1961;301:1-67.

6. Sorsby A, Leary GA. A longitudinal study of refraction and its components during growth. Spec Rep Ser Med Res Counc (G B). 1969;309:1-41.

7. Garner LF, Yap M, Scott R. Crystalline lens power in myopia. Optom Vis Sci. 1992;69:863-5.

8. Gordon RA, Donzis PB. Refractive development of the human eye. Arch Ophthalmol. 1985;103:785-9.

9. Jones LA, Mitchell GL, Mutti DO, et al. Comparison of ocular component growth curves among refractive error groups in children. Invest Ophthalmol Vis Sci. 2005;46:2317-27.

10. Twelker JD, Mitchell GL, Messer DH, et al. Children's ocular components and age, gender, and ethnicity. Optom Vis Sci. 2009;86:918-35.

11. Wong HB, Machin D, Tan SB, et al. Ocular component growth curves among Singaporean children with different refractive error status. Invest Ophthalmol Vis Sci. 2010;51:1341-7.

12. Saw SM, Chua WH, Hong CY, et al. Nearwork in early-onset myopia. Invest Ophthalmol Vis Sci. 2002;43:332-339.

13. Saw SM, Chua WH, Hong CY, et al. Height and its relationship to refraction and biometry parameters in Singapore Chinese children. Invest Ophthalmol Vis Sci. 2002;43:1408-1413.

14. Olsen T. On the calculation of power from curvature of the cornea. Br J Ophthalmol. 1986;70:152-4.

15. Bennett AG. A method of determining the equivalent powers of the eye and its crystalline lens without resort to phakometry. Ophthalmic Physiol Opt. 1988;8:53-9.

16. Mutti DO, Zadnik K, Adams AJ. The equivalent refractive index of the crystalline lens in childhood. Vision Res. 1995;35:156573. 
17. Saw SM, Carkeet A, Chia KS, et al. Component dependent risk factors for ocular parameters in Singapore Chinese children. Ophthalmology. 2002;109:2065-71.

18. Garner LF, Stewart AW, Owens H, et al. The Nepal Longitudinal Study: biometric characteristics of developing eyes. Optom Vis Sci. 2006;83:274-80.

19. Morgan IG, Rose KA, Ellwein LB; Refractive Error Study in Children Survey Group. Is emmetropia the natural endpoint for human refractive development? An analysis of populationbased data from the refractive error study in children (RESC). Acta Ophthalmol. 2010;88:877-84.

20. Morgan I, Rose KA. How genetic is school myopia? Prog Retin Eye Res. 2005;24:1-38.

21. Siegwart JT Jr, Norton TT. Perspective: how might emmetropization and genetic factors produce myopia in normal eyes? Optom Vis Sci. 2011;88:E365-72.

22. Wallman $\mathrm{J}$, Winawer $\mathrm{J}$. Homeostasis of eye growth and the question of myopia. Neuron. 2004;43:447-68.

23. van Alphen GWHM. On emmetropia and ametropia. Opbthalmologica Suppl. 1961;142:1-92.

24. Mutti DO, Zadnik K, Fusaro RE, et al. Optical and structural development of the crystalline lens in childhood. Invest Ophthalmol Vis Sci. 1998;39:120-33.

25. Zadnik K, Mutti DO, Fusaro RE, Adams AJ. Longitudinal evidence of crystalline lens thinning in children. Invest Ophthalmol Vis Sci. 1995;36:1581-7.

26. Brown NP, Koretz JF, Bron AJ. The development and maintenance of emmetropia. Eye. 1999;13:83-92.

27. Dubbelman M, Van der Heijde GL. The shape of the aging human lens: curvature, equivalent refractive index and the lens paradox. Vision Res. 2001;41:1867-77.

ED: Please verify the accuracy of any edits made to the article summary below.

Précis: Crystalline lens power calculations were made in children of the Singapore Cohort study Of the Risk factors for Myopia, showing that newly developed myopic children had greater decreases in lens power than persistent emmetropic children.
28. Pierscionek BK. Presbyopia-effect of refractive index. Clin Exp Optom. 1990;73:23-30.

29. Kasthurirangan S, Markwell EL, Atchison DA, Pope JM. In vivo study of changes in refractive index distribution in the human crystalline lens with age and accommodation. Invest Ophthalmol Vis Sci. 2008;49:2531-2540.

30. Hashemi H, Iribarren R, Morgan IG, et al. Increased hyperopia with ageing based on cycloplegic refractions in adults: the Tehran Eye Study. Br J Ophthalmol. 2010;94:20-3.

31. Augusteyn RC. On the growth and internal structure of the human lens. Exp Eye Res. 2010;90:643-54.

32. Mutti DO, Mitchell GL, Sinnott LT, et al. Corneal and crystalline lens dimensions before and after myopia onset. Optom Vis Sci. 2012;89:251-262.

33. Xiang F, He M, Morgan IG. Annual changes in refractive errors and ocular components before and after the onset of myopia in Chinese children. Ophthalmology. 2012;119:1478-1484.

34. Mutti DO, Hayes JR, Mitchell GL, et al. Refractive error, axial length, and relative peripheral refractive error before and after the onset of myopia. Invest Ophthalmol Vis Sci. 2007;48: 2510-9.

35. Dunne MC, Barnes DA, Royston JM. An evaluation of Bennett's method for determining the equivalent powers of the eye and its crystalline lens without resort to phakometry. Ophthalmic Physiol Opt. 1989;9:69-71.

36. Rozema JJ, Atchison DA, Tassignon MJ. Comparing methods to estimate the human lens power. Invest Ophthalmol Vis Sci. 2011;52:7937-42.

37. Bennett AG, Rabbetts RB. Clinical Visual Optics. Oxford, England: Butterworth-Heinemann Ltd: 1989;411. 